
Cahiers
de a Recherche
Forles Droits
Fondamentaux

\section{Cahiers de la recherche sur les droits} fondamentaux

$15 \mid 2017$

Le corps humain saisi par le droit : entre liberté et propriété

\title{
Le Protocole additionnel à la Convention du Conseil de l'Europe pour la prévention du terrorisme
}

The Additional Protocol to the Council of Europe Convention on the Prevention of Terrorism

Mamoud Zani

\section{OpenEdition}

Journals

Édition électronique

URL : https://journals.openedition.org/crdf/566

DOI : $10.4000 /$ crdf.566

ISSN : 2264-1246

Éditeur

Presses universitaires de Caen

Édition imprimée

Date de publication : 1 novembre 2017

Pagination : 127-132

ISBN : 978-2-84133-858-0

ISSN : 1634-8842

Référence électronique

Mamoud Zani, «Le Protocole additionnel à la Convention du Conseil de l'Europe pour la prévention du terrorisme », Cahiers de la recherche sur les droits fondamentaux [En ligne], 15 | 2017, mis en ligne le 01 octobre 2019, consulté le 14 novembre 2022. URL : http://journals.openedition.org/crdf/566 ; DOI : https://doi.org/10.4000/crdf.566 


\title{
Le Protocole additionnel à la Convention du Conseil de l'Europe pour la prévention du terrorisme
}

\author{
Mamoud ZANI \\ Professeur de droit public à la faculté de droit de Tunis (Tunisie) \\ Directeur du Centre de droit international et européen (CDIE) de Tunis
}

I. Les traits essentiels du Protocole

A. L'incrimination des actes terroristes

B. L'instauration des points de contact nationaux

II. Les mécanismes spécifiques d'application du Protocole
A. Le Comité d'experts sur le terrorisme
B. Le Comité des États parties

Le terrorisme ${ }^{1}$ est un phénomène complexe qui va au-delà de la sphère nationale. Depuis les attentats du World Trade Center sur le sol des États-Unis d'Amérique en $2001^{2}$, cet acte abject de terreur a pris une dimension tout à fait particulière; en effet, le terrorisme est devenu transnational comme en témoigne la vague d'attentats qui a frappé de plein fouet le monde tout entier et l'Europe en particulier: Indonésie (Bali, 2002), Maroc (Casablanca, 2003), Turquie (Istanbul, 2003), Espagne (Madrid, 2004), Arabie saoudite (Médine, Jeddah, Qatif, 2004), RoyaumeUni (Londres, 2005), France (Paris, 2015 et 2017; Nice, 2016), Belgique (Bruxelles, 2016), Canada (Québec, 2017), Royaume-Uni (Londres, 2017).
L'importance conférée par le Conseil de l'Europe à la question du terrorisme ne date point d'aujourd'hui. Effectivement, la prévention et la répression du terrorisme demeurent une préoccupation majeure de l'institution strasbourgeoise qui s'est dotée au fil du temps d'un arsenal normatif conséquent en la matière. Il sied à cet égard de mentionner, à titre d'exemple, la Convention pour la répression du terrorisme (1977); le Protocole portant amendement à la Convention pour la répression du terrorisme (2003); la Convention pour la prévention du terrorisme (2005) ; la Convention relative au blanchiment, au dépistage, à la saisie et à la confiscation des produits du crime et au financement du terrorisme (2005); le plan

1. Selon le Comité des ministres du Conseil de l'Europe, le terrorisme "porte gravement atteinte aux droits de l'homme, menace la démocratie, vise notamment à déstabiliser des gouvernements légitimement constitués et à saper la société civile pluraliste et remet en cause l'idéal des personnes à vivre libérées de la terreur» (Lignes directrices du Comité des ministres sur la protection des victimes d'actes terroristes, adoptées le 2 mars 2005, préambule, point a). Voir aussi Les droits de l'homme et la lutte contre le terrorisme. Les lignes directrices du Conseil de l'Europe, Strasbourg, Éditions du Conseil de l'Europe, 2005.

2. Voir L. Condorelli, «Les attentats du 11 septembre et leurs suites: où va le droit international?», Revue générale de droit international public, t. 105, $\mathrm{n}^{\circ}$ 4, 2001, p. 829-848; du même auteur, L'optimisme de la raison, Paris, A. Pedone, 2014; S. Sur, Le Conseil de sécurité dans l'après-11 septembre, Paris, LGDJ, 2004. 
d'action ${ }^{3}$ du Conseil de l'Europe pour lutter contre l'extrémisme violent et la radicalisation conduisant au terrorisme (2015), etc.

Pour préserver cet acquis normatif et parfaire sa quintessence, le Conseil de l'Europe mit en place en 2001 le Groupe multidisciplinaire sur l'action internationale contre le terrorisme (GMT) dont la fonction consistait essentiellement à examiner «la possibilité de mettre à jour les instruments internationaux existants au Conseil de l'Europe en matière de lutte contre le terrorisme ${ }^{4}$ et de préparer un rapport sur

[...] les actions qui pourraient être menées utilement par le Conseil de l'Europe dans le domaine de la lutte contre le terrorisme, en tenant compte des travaux réalisés dans d'autres enceintes internationales ([Organisation des Nations unies] et [Organisation pour la sécurité et la coopération en Europe] notamment) et au sein de l'Union européenne ${ }^{5}$,

outre le respect des valeurs sur lesquelles repose l'institution strasbourgeoise.

À la suite de l'expiration du mandat du Groupe multidisciplinaire sur l'action internationale contre le terrorisme, le 31 décembre 2002, le Comité des ministres du Conseil de l'Europe a mis en place un nouvel organe chargé de coordonner l'action du Conseil contre le terrorisme, à savoir le Comité d'experts sur le terrorisme (CODEXTER). Celui-ci est à l'origine de l'adoption par le Comité des ministres, le 3 mai 2005, de la Convention pour la prévention du terrorisme, dans le but

[...] d'améliorer les efforts des Parties dans la prévention du terrorisme et de ses effets négatifs sur la pleine jouissance des droits de l'homme et notamment du droit à la vie, à la fois par des mesures à prendre au niveau national et dans le cadre de la coopération internationale, en tenant compte des traités ou des accords bilatéraux et multilatéraux existants, applicables entre les Parties ${ }^{6}$.

Pour faire face au phénomène répandu des combattants terroristes étrangers et partant compléter l'instrument cité ci-avant, le Comité des ministres a institué, le 22 janvier 2015, le Comité sur les combattants terroristes étrangers et les questions connexes (COD-CTE), en vue de rédiger, sous la direction du CODEXTER, un projet de Protocole additionnel complétant la Convention du Conseil de l'Europe sur la prévention du terrorisme.

Dans le cadre du mandat qui lui a été assigné, le CODCTE devait, entre autres, prêter attention à la pénalisation d'un certain nombre d'actes:
- se faire recruter, ou tenter de se faire recruter, pour le terrorisme;

- recevoir, ou tenter de recevoir, un entraînement pour le terrorisme;

- se rendre, ou tenter de se rendre, dans un État autre que son État de résidence ou de nationalité, dans le but de commettre, d'organiser ou de préparer des actes de terrorisme, ou afin d'y participer ou de dispenser ou recevoir un entraînement au terrorisme;

- fournir ou collecter des fonds destinés à financer ces voyages;

- organiser et faciliter ces voyages (excepté «le recrutement pour le terrorisme») ${ }^{7}$.

La prise en compte de ces nouvelles formes de terrorisme s'inscrit indubitablement dans l'esprit de la résolution 2178 (2014) du Conseil de sécurité des Nations unies visant à éradiquer la menace des combattants terroristes étrangers, en l'occurrence:

[...] des individus qui se rendent dans un État autre que leur État de résidence ou de nationalité, dans le dessein de commettre, d'organiser ou de préparer des actes de terrorisme, ou afin d'y participer ou de dispenser ou recevoir un entraînement au terrorisme, notamment à l'occasion d'un conflit armé $[\ldots]^{8}$

en exigeant des États qu'ils veillent

[...] à ce que la qualification des infractions pénales dans leur législation et leur réglementation internes permette, proportionnellement à la gravité de l'infraction, d'engager des poursuites et de réprimer $[\ldots]$ :

- la fourniture ou la collecte délibérées, par quelque moyen que ce soit, directement ou indirectement, par leurs nationaux ou sur leur territoire, de fonds que l'on prévoit d'utiliser ou dont on sait qu'ils seront utilisés pour financer les voyages de personnes [susceptibles] de commettre [...] des actes de terrorisme;

- l'organisation délibérée, par leur nationaux ou sur leur territoire, des voyages de personnes qui se rendent dans un État autre que leur État de résidence ou de nationalité, dans le dessein de commettre, d'organiser ou de préparer des actes de terrorisme $[\ldots]^{9}$.

Dans le droit fil de l'action du Conseil de l'Europe contre le terrorisme, l'Union européenne (UE) ${ }^{10}$ et l'Organisation des Nations unies (ONU) ont pour leur part développé un corpus normatif assez diversifié en la matière. Il en est ainsi, par exemple, pour l'UE avec la décision du Conseil européen du 3 décembre 1998, conférant à Europol la mission de traiter les infractions commises ou susceptibles d'être commises dans le cadre

3. Comité des ministres, «Lutte contre l'extrémisme violent et la radicalisation conduisant au terrorisme», $125^{\mathrm{e}}$ session, Bruxelles, 19 mai 2015 , $\mathrm{CM}(2015) 74$-addfinal.

4. Comité des ministres, $109^{\mathrm{e}}$ session, Strasbourg, 7-8 novembre 2001, $\$ 5$.

5. Ibid.

6. Convention du Conseil de l'Europe pour la prévention du terrorisme, 16 mai 2005, STCE no 196, p. 2, art. 2.

7. Conseil de l'Europe, «Rapport explicatif du Protocole additionnel à la Convention du Conseil de l'Europe pour la prévention du terrorisme», 22 octobre 2015, STCE $n^{\circ} 217$, p. $2, \$ 7$

8. Conseil de sécurité des Nations unies, résolution 2178, 24 septembre 2014, S/RES/2178 (2014), p. 2, préambule.

9. Ibid., p. 5, principe 6.

10. Voir L'Union européenne et la lutte contre le terrorisme: état des lieux et perspectives, J. Auvret-Finck (dir.), Bruxelles, Larcier, 2010. 
d'activités terroristes portant atteinte à la vie, à l'intégrité physique, à la liberté des personnes et aux biens ; la recommandation du même organe en date du 9 décembre 1999 ayant trait à la coopération en matière de lutte contre le financement du terrorisme; la décision-cadre du Conseil du 13 juin 2002 relative à la lutte contre le terrorisme ${ }^{11}$ afin d'harmoniser les législations nationales en matière d'infractions terroristes; l'institution en mars $2004 \mathrm{du}$ poste de coordinateur de la lutte contre le terrorisme; la stratégie de l'UE de 2005 pour lutter contre le terrorisme reposant sur quatre principes d'actions: prévention, protection, poursuite et réaction; la communication de la Commission du 28 avril 2015 relative à l'élaboration du programme européen en matière de sécurité, pour la période 2015-2020.

Quant à l'ONU, il convient de mentionner les résolutions suivantes du Conseil de sécurité: 1267 (1999) exigeant des États parties à l'ONU le gel des avoirs des personnes ou des entités associées à Al-Qaïda et aux Taliban; 1373 (2001) mettant en place le Comité contre le terrorisme; 1540 (2004) exhortant les États à empêcher les acteurs non étatiques de mettre au point, de se procurer, de fabriquer, de posséder, de transporter ou de transférer des armes nucléaires, chimiques ou biologiques; 1624 (2005) invitant les États à adopter des mesures pour prohiber l'incitation à commettre des actes terroristes, prévenir une telle incitation et refuser l'asile à toute personne impliquée à cet effet; 2195 (2014) préconisant une action collective en vue de prévenir et combattre le terrorisme sous toutes ses formes et dans toutes ses manifestations, y compris le terrorisme associé à la criminalité transnationale organisée. Ajoutons la Stratégie antiterroriste mondiale mise en place par l'Assemblée générale de l'ONU, le 8 septembre $2006^{12}$, ainsi que l'Équipe spéciale de lutte contre le terrorisme (2005) chargée de coordonner les actions de l'organisation au sein du système des Nations unies.

En vue de compléter l'ensemble de ces efforts et de conforter davantage son action en faveur de la prévention et de la répression du terrorisme, notamment contre le phénomène des combattants terroristes étrangers ${ }^{13}$ menaçant au quotidien la sécurité des États européens, le Comité d'experts sur le terrorisme a soumis, le 10 avril 2015, au Comité des ministres du Conseil de l'Europe le projet de Protocole additionnel à la Convention pour la prévention du terrorisme. À l'invitation de l'organe exécutif de l'institution strasbourgeoise, l'Assemblée parlementaire a approuvé, le 23 avril 2015, l'avis no 289 sur ledit projet tout en recommandant de renforcer le texte de celui-ci par les garanties de respect des droits de l'homme ${ }^{14}$. En définitive, le Protocole additionnel à la Convention du Conseil de l'Europe pour la prévention du terrorisme a été adopté par le Comité des ministres, lors de sa $125^{\mathrm{e}}$ session tenue à Bruxelles (Belgique), le 19 mai 2015, et ouvert à la signature des États parties à Riga (Lettonie), le 22 octobre 2015.

L'intérêt d'examiner cet instrument qui n'est pas encore en vigueur ${ }^{15}$, dédié à la question des combattants terroristes étrangers à la lumière de la résolution 2178 (2014) du Conseil de sécurité des Nations unies, consiste à supputer sa portée par rapport au cadre normatif précité. De manière concrète, il sied de se poser la problématique suivante: le Protocole additionnel constitue-t-il une véritable panacée pour venir à bout du phénomène des combattants terroristes étrangers?

Pour ce faire, nous examinerons, tout d'abord, les traits essentiels du Protocole (I), puis les mécanismes consacrés à son application (II).

\section{Les traits essentiels du Protocole}

L'originalité du Protocole additionnel à la Convention du Conseil de l'Europe pour la prévention du terrorisme repose sur deux aspects: d'un côté, le Protocole érige certains actes de terrorisme en infractions pénales (A), de l'autre, il établit des points de contact nationaux (B) pour faciliter l'échange d'informations entre les États parties.

\section{A. L'incrimination des actes terroristes}

À l'instar de la Convention pour la prévention du terrorisme qualifiant d'infractions pénales un certain nombre de conduites susceptibles d'aboutir à la commission d'actes terroristes, telles que la provocation publique ${ }^{16}$, le recrutement et l'entraînement ${ }^{17}$, le Protocole ajoute d'autres catégories d'infractions « de nature préparatoire par rapport à des actes terroristes ${ }^{18}$.

Ces infractions prévues aux articles 2 à 6 du Protocole sont au nombre de quatre, à savoir :

- participer aux activités d'une association ou d'un groupe en vue d'accomplir ou de prendre part à la commission d'une ou de plusieurs infractions terroristes par l'association ou le groupe (art. 2). À l'origine,

11. Voir M.-A. Beernaert, «La décision-cadre du 13 juin 2002 relative à la lutte contre le terrorisme», Revue internationale de droit pénal, vol. 77, $n^{\circ} 1-2,2006$, p. 277-284.

12. Doc. A/RES/6o/288

13. Voir la recommandation 2084 (2016) et la résolution 2091 (2016) de l'Assemblée parlementaire du Conseil de l'Europe sur «Les combattants étrangers en Syrie et en Irak».

14. Voir l'avis $n^{\circ} 289$ de l'Assemblée parlementaire du Conseil de l'Europe du 23 avril $2015, \$ 5$.

15. Au 28 mars 2017, le Protocole enregistre trente signatures et cinq ratifications. L'article 10, alinéa 2 du Protocole exige six instruments de ratification pour son entrée en vigueur.

16. Convention du Conseil de l'Europe pour la prévention du terrorisme, p. 3, art. 5 .

17. Ibid., p. 3, art. 6 et 7 .

18. Conseil de l'Europe, «Rapport explicatif du Protocole additionnel...», p. 2, §10. 
les rédacteurs du Protocole devaient procéder à l'examen de l'incrimination de l'acte de « recrutement pour le terrorisme» envisagé par l'article 6 de la Convention pour la prévention du terrorisme, mais au final cette proposition n'a pu être retenue car «l'incrimination d'un comportement "passif" ("se faire recruter pour le terrorisme") créerait des problèmes dans certains systèmes juridiques ${ }^{19}$;

- recevoir un entraînement pour le terrorisme (art. 3) $)^{20}$ à travers des instructions et l'obtention des connaissances ou des compétences pratiques de la part d'une autre personne pour fabriquer ou utiliser des explosifs, des armes à feu ou d'autres armes ou substances nocives ou dangereuses, d'autres méthodes ou techniques spécifiques, dans le but de commettre une infraction terroriste ou de contribuer à sa commission.

En incluant cette infraction, les rédacteurs du Protocole n'ont pas jugé opportun de retenir «le simple fait de visiter des sites Internet contenant des informations ou de recevoir des communications ${ }^{21}$ pouvant être utilisées pour s'entraîner au terrorisme au motif qu'il soit insuffisant pour former l'infraction en question. Il en est de même quant à l'incrimination de «la tentative ou la complicité ${ }^{22}$ se rapportant à celle-ci laissant ainsi la question au pouvoir d'appréciation des États sur la possibilité d'incorporer dans leurs ordres juridiques nationaux une telle sanction;

- se rendre à l'étranger à des fins de terrorisme comprend l'acte de se rendre dans un État autre que celui de l'État de nationalité ou de résidence habituelle du voyageur, en perspective de commettre, de contribuer ou de participer à une infraction terroriste (art. 4 , al. $\left.1^{\mathrm{er}}\right)$. Cette infraction s'inspire du paragraphe 6 , alinéa a, de la résolution $2178(2014)^{23}$ du Conseil de sécurité des Nations unies obligeant les États à engager des poursuites et à réprimer leurs nationaux qui se rendent ou tentent de se rendre dans un État étranger pour commettre, organiser ou préparer des actes de terrorisme. Tenant compte du principe de la liberté de circulation des personnes ${ }^{24}$, l'article 4 du Protocole ne prohibe pas de manière générale et n'incrimine pas tous les types de voyages. Dans une optique similaire, les rédacteurs du Protocole n'ont pas retenu l'incrimination de la complicité ${ }^{25}$ de cette infraction, hormis la tentative de voyager; celle-ci doit être réprimandée en application de la législation nationale comme «un acte préparatoire ou une tentative de commettre l'infraction terroriste principale ${ }^{26}$. Par ailleurs, tout acte visant à organiser ou faciliter la commission de l'infraction décrite dans l'article 4, alinéa 1 doit être sanctionné, en vertu de l'article 6, alinéa 1 du Protocole.

- financer des voyages à l'étranger par la fourniture ou la collecte de fond ${ }^{27}$ permettant totalement ou partiellement à toute personne de se rendre à l'étranger à des fins de terrorisme (art. 5 , al. $\left.1^{\text {er }}\right)$. Cette infraction est reprise du paragraphe 6 , alinéa $b$ de la résolution 2178 (2014) du Conseil de sécurité exigeant des États parties qu'ils sanctionnent:

[...] la fourniture ou la collecte délibérées, par quelque moyen que ce soit, directement ou indirectement, par leurs nationaux ou sur leur territoire, de fonds que l'on prévoit d'utiliser ou dont on sait qu'ils seront utilisés pour financer les voyages de personnes qui se rendent dans un État autre que leur État de résidence ou de nationalité $[\ldots]^{28}$.

Par rapport à la nature des fonds, ceux-ci

[...] peuvent provenir d'une source unique, par exemple un prêt ou un don accordé au voyageur par une personne physique ou morale, ou de sources diverses au moyen d'une forme de collecte organisée par une ou plusieurs personnes physiques ou morales ${ }^{29}$.

Les États parties au Protocole sont tenus de prendre les mesures nécessaires au plan national pour ériger en infractions pénales ${ }^{30}$ les manquements mentionnés dans les articles 2 à $6 \mathrm{du}$ Protocole, lorsque ceux-ci sont commis illégalement et intentionnellement. En procédant ainsi, ils sont obligés, d'une part, d'assurer le respect des libertés énoncées dans la Convention de sauvegarde des droits de l'homme et des libertés fondamentales de 1950, à savoir la liberté de circulation, la liberté d'expression, la liberté d'association et la liberté de religion; d'autre part, de prendre en considération le principe de proportionnalité ${ }^{31}$

19. Conseil de l'Europe, «Rapport explicatif du Protocole additionnel...», p. 5, $\$ 31$.

20. Cette infraction est reprise de l'article 7 , alinéa $1^{\text {er }}$ de la Convention pour la prévention du terrorisme.

21. Conseil de l'Europe, «Rapport explicatif du Protocole additionnel... », p. 6, \$ 40.

22. Ibid., p. 6, $\$ 42$.

23. Conseil de sécurité des Nations unies, résolution 2178, p. $5, \$ 6$. a.

24. Conseil de l'Europe, Protocole $\mathrm{n}^{\circ} 4$ à la Convention de sauvegarde des droits de l'homme et des libertés fondamentales, Strasbourg, 16 septembre 1963 , STCE $n^{\circ} 46$, p. 1 , art. 2.

25. Il appartient aux États parties de l'incorporer dans leur législation interne.

26. Conseil de l'Europe, «Rapport explicatif du Protocole additionnel...», p. 8, §53.

27. Suivant l'article 1 , alinéa 1 de la Convention internationale pour la répression du financement du terrorisme adoptée par l'Assemblée générale de l'ONU, le 9 décembre 1999, cette notion «s'entend des biens de toute nature, corporels ou incorporels, mobiliers ou immobiliers, acquis par quelque moyen que ce soit, et des documents ou instruments juridiques sous quelque forme que ce soit, y compris sous forme électronique ou numérique, qui attestent un droit de propriété ou un intérêt sur ces biens, et notamment les crédits bancaires, les chèques de voyage, les chèques bancaires, les mandats, les actions, les titres, les obligations, les traites et les lettres de crédit, sans que cette énumération soit limitative».

28. Conseil de sécurité des Nations unies, résolution 2178, p. $5, \$ 6 . b$.

29. Conseil de l'Europe, «Rapport explicatif du Protocole additionnel...», p. 9, \$56.

30. Voir les $\$ 2$ des articles 2 à 6 du Protocole.

31. Art. 8 , al. 2 du Protocole («Conditions et sauvegardes»): «Eu égard aux buts légitimes poursuivis et à leur nécessité dans une société démocratique ». 
et de proscrire «toute forme d'arbitraire, de traitement discriminatoire ou raciste $»^{32}$.

Ces obligations s'inspirent largement des dispositions de l'article 12 de la Convention pour la prévention du terrorisme visant à renforcer davantage la lutte contre le terrorisme tout en garantissant la protection des droits de l'homme et des libertés fondamentales.

\section{B. L'instauration des points de contact nationaux}

La particularité du Protocole additionnel à la Convention pour la prévention du terrorisme se traduit aussi par la mise en place des points de contact nationaux, en vue d'échanger des informations ${ }^{33}$ entre les États parties pour ce qui est des personnes se rendant à l'étranger à des fins de terrorisme. À cet effet, chaque État partie doit désigner un point de contact disponible vingt-quatre heures sur vingtquatre, sept jours sur sept doté des moyens nécessaires pour correspondre de manière permanente selon une procédure accélérée ${ }^{34}$ avec les autres points de contact des autres parties contractantes.

L'article 7 du Protocole relatif à l'échange d'informations policières s'explique par l'appel lancé aux États membres de l'ONU par le Conseil de sécurité, dans sa résolution 2178 (2014), afin d'intensifier et d'accélérer

[...] les échanges d'informations opérationnelles au sujet des activités ou des mouvements de terroristes et de réseaux terroristes, y compris de combattants terroristes étrangers, notamment avec les États de résidence ou de nationalité des individus concernés $[\ldots]^{35}$.

Il s'inspire à plein des dispositions de l'article 35 de la Convention du Conseil de l'Europe sur la cybercriminalité ${ }^{36}$ adoptée à Budapest (Hongrie), le 23 novembre 2001. Le paragraphe 1 de cet article impose à chaque État partie de désigner un point de contact dans le but d'assurer une assistance immédiate pour des investigations ayant trait aux infractions pénales relatives à des systèmes et à des données informatiques, ou pour collecter les preuves sous forme électronique d'une infraction pénale. Cette assistance concerne l'apport de conseils techniques et d'informations à caractère juridique, la conservation des données et la localisation des suspects. Pour faciliter le fonctionnement harmonieux du réseau, chaque État partie doit disposer d'un personnel qualifié, formé et équipé.

Les traits caractérisant le Protocole additionnel à la Convention pour la prévention du terrorisme ne suffisent pas à supputer la portée de l'instrument européen, il importe donc d'examiner les mécanismes consacrés à sa mise en œuvre.

\section{Les mécanismes spécifiques d'application du Protocole}

La Convention pour la prévention du terrorisme prévoit un comité conventionnel, en l'occurrence la Consultation des États parties chargée de l'application de ses dispositions dans les ordres juridiques nationaux. Par conséquent, il est raisonnable que le Protocole ajouté à cet instrument ne puisse pas envisager un tel mécanisme.

Le contrôle de l'application des principes du Protocole relève de la compétence du Comité d'experts sur le terrorisme (A), ainsi que de celle du Comité des États parties (B).

\section{A. Le Comité d'experts sur le terrorisme}

Le Comité d'experts sur le terrorisme (CODEXTER) ${ }^{37}$ est un rouage essentiel du Conseil de l'Europe en matière de terrorisme. Il lui appartient d'assurer le suivi des instruments juridiques antiterroristes ${ }^{38}$ de l'institution strasbourgeoise et de coordonner ses actions dans la lutte contre le terrorisme.

Composé des représentants des États membres, le CODEXTER est principalement chargé du contrôle des normes du Conseil de l'Europe afférentes à la lutte contre le terrorisme, en particulier l'application in concreto de la Convention pour la prévention du terrorisme. Pour ce faire, le comité procède à l'examen des profils nationaux contenant des informations sur les législations et les politiques nationales se rapportant à la lutte contre le terrorisme; ainsi que l'état des signatures et ratifications des instruments pertinents dans le domaine. De surcroît, il assure la promotion effective de ceux-ci en tenant compte des expériences significatives s'agissant de la lutte contre le terrorisme dans le respect des droits de l'homme et de l'État de droit, coordonne les activités de l'ensemble des comités intergouvernementaux dans leur action contre le terrorisme, et propose à l'organe exécutif du Conseil de l'Europe les mesures nécessaires pour renforcer la politique de lutte contre le terrorisme.

Dans ce sens, le CODEXTER s'intéresse ${ }^{39}$ à la question d'Internet et le terrorisme, les rapports entre ce dernier et le crime organisé, les procédures spéciales d'enquête et les insuffisances du cadre juridique international dans le domaine de la prévention et de la répression du terrorisme.

32. Ibid.

33. Voir l'article 7 du Protocole.

34. Le Protocole reste muet sur le fonctionnement de cette procédure.

35. Conseil de sécurité des Nations unies, résolution 2178, p. $4, \S_{3}$.

36. Conseil de l'Europe, Convention sur la cybercriminalité, Budapest, 23 novembre 2001 , STCE no 185 .

37. Institué par le Comité des ministres conformément à l'article 17 du Statut du Conseil de l'Europe et à la résolution CM/Res (2011) 24 concernant les comités intergouvernementaux et les organes subordonnés, leur mandat et leurs méthodes de travail.

38. Voir les instruments cités en introduction.

39. Selon la feuille de route du Comité pour l'exercice 2016-2017: CODEXTER (2016) OJ2 prov, Strasbourg, 10 novembre 2016 


\section{B. Le Comité des États parties}

Le Comité ou la Consultation des États parties est un mécanisme souple ${ }^{40}$ chargé du contrôle de l'application de la Convention pour la prévention du terrorisme. Il comprend tous les États parties et est convoqué à l'initiative du secrétaire général du Conseil de l'Europe, à la demande du Conseil des ministres ou de la majorité des États parties ${ }^{41}$.

Le rôle essentiel du Comité consiste à faciliter l'échange d'informations sur les développements juridiques, politiques ou techniques importants en matière de terrorisme; à formuler des propositions visant à améliorer l'usage et la mise en œuvre effectifs de la Convention; à émettre un avis sur toute question relative à la mise en œuvre de la Convention et sur toute proposition d'amendement de celle-ci; à examiner tout refus lié à une demande d'extradition présentée par un État partie ${ }^{42}$. Pour mener à bien sa tâche de contrôle, le comité est assisté d'un secrétariat administratif.

Dans le même ordre d'idées, il faut remarquer que la Convention européenne pour la répression du terrorisme $^{43}$ envisage le Comité européen pour les problèmes criminels $^{44}$, afin d'en assurer l'application et le règlement amiable de tout problème résultant de son exécution. À la suite de l'adoption du Protocole portant amendement à la Convention pour la répression du terrorisme, le
15 mai 2003, la fonction du Comité a été élargie ${ }^{45}$, lui donnant la possibilité d'adresser au Comité des ministres des recommandations concernant les propositions d'amendements et l'adhésion des États non membres, de lui soumettre un rapport sur l'application des dispositions de la Convention au niveau interne et de formuler à la demande de tout État partie un avis consultatif s'agissant de l'application de celle-ci.

Le Protocole additionnel à la Convention européenne pour la prévention du terrorisme constitue à coup sûr un instrument ambitieux pour consolider le système du Conseil de l'Europe en matière de prévention et de répression des actes terroristes ${ }^{46}$ et partant édifier à l'échelle européenne un droit commun de lutte contre le fléau des combattants terroristes étrangers. Du reste, c'est sur ce point que l'instrument en question doit être apprécié quant à sa valeur ajoutée par rapport à la Convention sur la prévention du terrorisme ${ }^{47}$. D'autant plus que le Protocole fait peser sur les États parties à l'institution strasbourgeoise une obligation stricte pour ce qui est de son application, en l'occurrence le respect des droits de l'homme et de l'État de droit.

En dépit de l'enjeu du Protocole, sa portée et son efficacité par rapport à l'objectif escompté dépendent entièrement de la coopération des États et de la transposition de ses dispositions dans les systèmes pénaux nationaux des États parties.

40. L'article 30 de la Convention ne prévoit aucune condition temporelle pour sa convocation.

41. Convention du Conseil de l'Europe pour la prévention du terrorisme, p. 13, art. 30, al. 2.

42. Ibid., p. 13, art. 30, al. $1^{\text {er }}$.

43. Convention européenne pour la répression du terrorisme, adoptée à Strasbourg le 27 janvier 1977 et entrée en vigueur le 4 août 1978 , STCE ${ }^{\circ} 90$. Voir C. Vallée, «La Convention européenne pour la répression du terrorisme», Annuaire français de droit international, vol. 22, nº 1, 1976, p. 756-786.

44. Ibid., art. 9 .

45. Voir l'article 6 du Protocole portant amendement à la Convention européenne pour la répression du terrorisme, Strasbourg, 15 mai 2003 , STCE $\mathrm{n}^{\circ} 190$.

46. Suivant la recommandation 1426 (1999) de l'Assemblée parlementaire du Conseil de l'Europe («Démocraties européennes face au terrorisme»), il faut comprendre par acte terroriste « tout délit commis par des individus ou des groupes recourant à la violence ou menaçant de l'utiliser contre un pays, ses institutions, sa population en général ou des individus concrets, qui, motivé par des aspirations séparatistes, par des conceptions idéologiques extrémistes ou par le fanatisme, ou inspiré par des mobiles irrationnels et subjectifs, vise à soumettre les pouvoirs publics, certains individus ou groupes de la société, ou d'une façon générale, l'opinion publique à un climat de terreur» $(\$ 5)$.

47. Cet instrument prévoit de manière originale une disposition relative à la protection et à l'indemnisation des victimes du terrorisme (Convention du Conseil de l'Europe pour la prévention du terrorisme, p. 5, art. 13). 